\title{
The Role of Experiential Avoidance in Terms of Fatigue and Pain During COVID-19 Among Latinx Adults
}

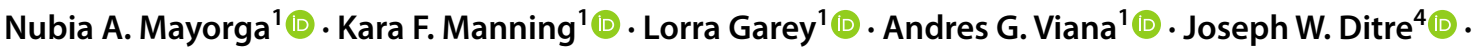 \\ Michael J. Zvolensky ${ }^{1,2,3,5}$
}

Accepted: 17 January 2022 / Published online: 31 January 2022

○ The Author(s), under exclusive licence to Springer Science+Business Media, LLC, part of Springer Nature 2022

\begin{abstract}
Background Latinx persons are overrepresented in terms of 2019 Coronavirus (COVID-19) infection rates and constitute a subpopulation at increased risk for COVID-19 related physical ailments. Fatigue and pain are among the most prevalent somatic symptoms among the Latinx population; however, there is little understanding of individual difference factors that are related to fatigue and pain during COVID-19 among this health disparities population. Experiential avoidance (EA) reflects the persistent tendency to avoid aversive internal sensations.

Methods The current study sought to extend past work by exploring EA in relation to fatigue severity, pain intensity, and pain disability among 182 Latinx adult persons during the COVID-19 pandemic.

Results Results indicated that EA accounted for a statistically significant amount of variance across the criterion variables. Conclusions Overall, the current work provides initial empirical evidence that EA is related to greater fatigue severity and pain severity/disability among Latinx persons during COVID-19.
\end{abstract}

Keywords COVID-19 $\cdot$ Latinx $\cdot$ Fatigue $\cdot$ Pain $\cdot$ Experiential avoidance

\section{Introduction}

The 2019 Coronavirus (COVID-19) pandemic has had a devastating global impact, resulting in upwards of 105 million infections and over 2.3 million deaths (February 2021; WHO Coronavirus Disease (COVID-19) Dashboard). Within the United States (U.S.), COVID-19 infection rates have been reported as high as 26 million (CDC, 2020). Importantly, racial, and ethnic disparities have been reported in terms of COVID-19 infection rates and mortality in the U.S. (Resnick

Michael J. Zvolensky

mjzvolen@central.uh.edu

1 Department of Psychology, University of Houston, 126 Heyne Building, Houston, TX, USA

2 Department of Behavioral Science, The University of Texas MD Anderson Cancer Center, Houston, TX, USA

3 HEALTH Institute, University of Houston, Houston, TX, USA

4 Department of Psychology, Syracuse University, New York, NY, USA

5 Department of Psychology, University of Houston, 3695 Cullen Blvd., Room 126, Houston, TX 77204, USA et al., 2020). Indeed, despite accounting for approximately $18.3 \%$ of the U.S. population, Center for Disease Control and Prevention (CDC) weighted population distributions indicate that Latinx persons comprise 33.8\% of COVID-19 mortality cases (COVID-19 Provisional Counts-Health Disparities, 2021). Further, even without COVID-19 infection, pandemic-related stress may be an especially salient source of increased physical distress among Latinx persons (Zvolensky et al., 2020a). Thus, there is an urgent public health need to identify what possible individual difference factors may be related to physical health problems among Latinx persons in the context of COVID-19.

COVID-19 research has identified certain physical symptoms have increased across the general population among those with and without COVID-19 infection (Zerbe, 2020). Fatigue, defined as an overwhelming sense of tiredness and lack of energy, has been identified as one prominent and prevalent symptom among COVID-19 survivors, (Wright \& O'Connor, 2014) with approximately $64 \%$ of persons within the general population reporting experiencing severe fatigue related to the disease (Morgul et al., 2020). Fatigue irrespective of COVID-19 infection is also related to increased psychological distress (Meichtry et al., 2020; Morgul et al., 
2020; Zerbe, 2020). However, little is known about the experience of fatigue among Latinx persons, specifically, during the COVID-19 pandemic. Of the available non-COVID-19 research, studies from community samples have found higher levels of fatigue and chronic fatigue syndrome among Latinx adults when compared to non-Latinx Whites (Jason et al., 1999; Song et al., 1999). Various sociocultural factors have been associated with fatigue severity, including English (versus Spanish) speaking, greater age, lower income, female (versus male) sex, and acculturation among both general Latinx samples (Chen, 1986; Loge et al., 1998) and chronic fatigue samples (Torres-Harding et al., 2008). There is clinical importance in evaluating factors related to fatigue among Latinx persons during COVID-19, as such symptoms can be related to poorer mental and physical health problems (Wright \& O'Connor, 2014) and lower the body's ability to fight infection (McKinney et al., 2015; Too Exhausted to Fight, Immune System May Harm the Body They Are Supposed to Protect-ScienceDaily).

Another prevalent health problem associated with the COVID-19 pandemic is the experience of pain (Who-ChinaJoint-Mission-on-Covid-19-Final-Report.Pdf, 2020), as several studies have found elevated rates of myalgia among COVID-19 survivors even among those without prior chronic pain issues (Kemp et al., 2020). Among the general population, COVID-19 pandemic has led to an increase of pain symptoms and severity of pain (Rogers et al., 2021). Investigating pain among Latinx persons specifically during COVID-19 is highly relevant to public health, as some non-COVID-19 work suggests that Latinx individuals may be more sensitive to pain than non-Latinx Whites and nonLatinx Blacks (Kwok \& Bhuvanakrishna, 2014; ReyesGibby et al., 2012). Several factors have been related to greater pain and disability among the Latinx population, including racial/ethnic discrimination (Bakhshaie et al., 2019), and substance use (Paulus et al., 2017). However, no research has focused on better understanding the experience of pain severity and pain disability among the general population of Latinx persons in the context of the COVID19 pandemic. By better understanding factors related to increased pain and pain disability among Latinx persons during COVID-19, there may be opportunity to intervene to reduce pain- and fatigue-related inequities among this group.

There is a public health need to investigate individual difference factors that may be related to the experience of fatigue and pain among Latinx persons in the context of the COVID-19 pandemic to inform intervention efforts. Past and current work has highlighted the tendency for individuals to avoid emotional and physical distress symptoms during large scale disasters (Lovell et al., 2020; Siebenhaar et al., 2020; Sprang \& LaJoie, 2009). One theoretically relevant individual difference factor to explore during COVID-19 is experiential avoidance (EA). EA reflects an individual's rigidly attempt to avoid aversive thoughts, feelings, physical sensations, and other internal experiences (Hayes et al., 1996). EA is theorized to lead to increased salience and functional importance of the avoided experiences (Hayes et al., 1996, 2004). In fact, avoidance is related to increased risk of more severe physical symptoms and psychological distress (Aldrich et al., 2000; McCracken \& Eccleston, 2003). Research among individuals with chronic pain suggests that attempting to control or avoid aversive internal symptoms, such as pain and fatigue, is associated with elevated psychological distress and physical disability (Aldrich et al., 2000; McCracken \& Eccleston, 2003). Moreover, higher EA has been related to lower pain threshold and slower recovery from acute pain (Feldner et al., 2008). Among individuals with chronic fatigue syndrome, lower EA has been related to lesser fatigue and psychological distress (Jacobson et al., 2001; Van Damme et al., 2006). Non-COVID-19 research among the general Latinx population has indicated that EA is an important psychological difference factor for increased pain severity and disability (Bakhshaie et al., 2019; Campbell et al., 2009). This work, however, has not yet been extended to experiences of fatigue and pain among Latinx persons during the COVID-19 pandemic.

The current study examined the role of EA in terms of pain severity and disability and fatigue among a general population sample of Latinx adults during the COVID-19 pandemic, after adjusting for theoretically and empirically relevant sociodemographic and COVID-19 related variables. Specifically, we adjusted for the following variables: years living in the U.S. (as this variable has been related to worse psychological functioning among Latinx persons; Velasco-Mondragon et al., 2016); age and education (those of younger age and higher level of education are purported to have better health and psychological functioning than those of older age and lower education; Lawrence, 2017); and, number of health conditions and work and life stress due to COVID-19, to account for general health (as it may impact fatigue and pain symptom severity as well as the exacerbation of physical symptoms due to COVID-19 stress; Koppert et al., 2021; Rogers et al., 2021). We predicted EA would be related to more severe fatigue, pain intensity, and pain disability during the COVID-19 pandemic over and above the variance accounted for by the covariates.

\section{Methods}

\section{Participants}

The present study included 182 Latinx persons $(32.4 \%$ female, $M_{\text {age }}=34.1$ years, $S D=8.25$ ). In line with social distancing guidelines, participants were recruited via an online survey panel program, Amazon Mechanical Turk (Mturk). 
Study eligibility criteria included being 18-65 years old, self-identifying as a Latinx person, currently living in the U.S., and being able to provide informed consent. Exclusion criteria included being younger than 18 years or older than 65 years, being a non-English speaker (to ensure comprehension of the study questions), and inability to give informed, and voluntary consent.

The present sample consisted of predominately Latinx White/Caucasian (52.2\%) individuals, followed by $26.9 \%$ Latinx Black/African American, 6.6\% identified as other, $6.0 \%$ as more than one race, 3.3\% Latinx Asian, 1.6\% Latinx Native American/Alaska Native, $1.6 \%$ as Latinx Native Hawaiian or Pacific Islander, and $1.6 \%$ declined to state. Most participants (65.4\%) identified as heterosexual, 29.7\% identified as bisexual, $2.7 \%$ identified as gay or lesbian, and $2.2 \%$ indicted that they preferred not to disclose their sexuality. Regarding education, $4.9 \%$ of the participants indicated some college, $4.9 \%$ had an associate degree, $56.0 \%$ had a bachelor's degree, $39.7 \%$ had a master's degree, and $1.1 \%$ had a doctoral degree. Most of the sample indicated they were married or living with someone (83.5\%). In terms of unemployment, $32.6 \%$ of the sample reported being laidoff or let go from their employment since the onset of the COVID-19 pandemic. Further $84.2 \%$ of the sample reported having a child in the home that could not attend school and $73.4 \%$ of persons reported having difficulty acquiring childcare when needed.

In terms of preexisting illnesses, $34.1 \%$ of persons reported having high blood pressure $33.0 \%$ diabetes, $25.8 \%$ autoimmune disease, $22.0 \%$ respiratory disease, $20.9 \%$ asthma, $20.3 \%$ gastrointestinal issues, $15.9 \%$ cardiovascular disease, $13.2 \%$ kidney disease, and 8.8\% HIV. Participants reported living in the U.S. an average of $31.14(S D=10.74)$ years and $96 \%$ of the sample indicated that they were born in the U.S.

In terms of fatigue severity, the average level of fatigue severity in the sample was $4.78(S D=1.43)$ (Krupp et al., 1989). Previous work has indicated an FSS score above 5 indicates "high level" fatigue (Bakshi et al., 1999; Lerdal et al., 2005; Manning et al., 2019). In addition, the average level of pain intensity was $21.47(S D=11.09)$ and the average level of pain disability was $16.76(S D=8.62)$. These scores are characteristic of high intensity pain using normative population data (Von Korff et al., 1992).

\section{Measures}

\section{Demographics Questionnaire}

Demographic information was gathered to characterize the sample and included age, gender, sexual orientation, primary language, ethnicity, education (recoded as $0=$ less than college degree and 1=Bachelor's degree or above), employment status, marital status, years in U.S., COVID19 impact (i.e., "How much has/did COVID-19 impacted your day-to-day life?"), COVID-19 exposure (i.e., "What is the approximate size of the COVID-19 outbreak in your area?"), as well as preexisting medical conditions. The COVID-19 impact question was scored on a 5-item Likert type scale with the following options: $1=$ not at all, $2=\mathrm{a}$ little, $3=$ much, $4=$ very much, $5=$ extremely.

\section{The Epidemic-Pandemic Impacts Inventory (EPII; Grasso et al., 2020)}

The EPII is a 92-item inventory of pandemic-related experiences that range across five life domains, including work life (12-items), home life (19-items), social activities and isolation (18-items), emotional/physical health and infection (24-items), and positive change (19-items). Each item has the response options of "Yes, Me", "Yes, Person in the Home", "No", and "Not Applicable". For scoring purposes, the categories that indicated a positive response (i.e., Yes) were consolidated into a value of 1 and the options indicated a negative response (i.e., No, Not applicable) were given a value of 0 . A total score was calculated by combining the positive values and a higher score was indicative of worse pandemic-related stress experiences within each domain (Grasso et al., 2020). The current study included work life (e.g., "hard time making the transition to working from home") and home life (e.g., "increase in physical conflict with a partner or spouse") as covariates in the current study (work: $\alpha=.88$, home: $\alpha=.89$ ).

\section{Brief Experiential Avoidance Questionnaire (BEAQ; Gamez et al., 2014)}

The BEAQ is a 15-item self-report measure that assesses six dimensions of EA including behavioral avoidance, distress aversion, procrastination, distraction/suppression, repression/denial, and distress endurance. All items are rated on a 6-point Likert scale ranging from 1 (strongly disagree) to 6 (strongly agree). The total score was used as a predictor variable and demonstrated excellent internal consistency $(\alpha=0.94)$.

\section{Fatigue Severity Scale (FSS; Krupp et al., 1989)}

The Fatigue Severity Scale is a psychometrically sound 9-item measure of fatigue severity. Items are rated on a 7-point Likert scale, ranging from 1 (no impairment) to 7 (severe impairment). Scores of 5 or higher indicate clinically significant levels of fatigue (Bakshi et al., 1999). The FSS in the current sample demonstrated excellent internal consistency $(\alpha=0.95)$ and was utilized as a criterion variable. 


\section{Graded Chronic Pain Scale (GCPS; Von Korff et al., 1992)}

The Graded Chronic Pain Scale is an 8-item self-report measure of pain that encompasses two subscales: (1) pain intensity and (2) pain disability. The pain intensity subscale includes items related to self-reported current pain, worst pain, and usual pain. Pain disability includes items that investigate to what degree the individual's daily functioning is limited by their pain. Participants are asked to rate their pain intensity on a scale from 0 (No pain) to 10 (Pain as bad as could be) and their pain disability from 0 (no interference) 10 (unable to carry on activities). With a range from 0 to 30 , higher scores indicate a greater pain intensity. In the current study, the pain intensity and pain disability subscales were used as criterion variables and demonstrated excellent internal consistency ( $\alpha=0.93$ and 0.92 ).

\section{Procedure}

Participants were recruited from across the U.S. via Amazon Mechanical Turk (Mturk) between June 2020 and November 2020; a time during the COVID-19 pandemic. Mturk is an online survey management system that has been found to yield valid and representative data (Kim \& Hodgins, 2017). Interested and eligible participants provided informed consent and completed the approximately 60-min online survey. Participants were compensated a total of $\$ 4.00$ for completing the survey with credit through their Mturk account. Mturk allows researchers to specify the quality criteria for their sample. In the current study, participants were shown the study information if they: (1) had never been blocked from a study, (2) performed at more than $90 \%$ accuracy on other studies, and (3) passed a Human Intelligence Test (HIT), such as identifying pictures or filling in a CAPTCHA test. To further ensure quality of the data, IP addresses were obtained to prohibit duplicate responses and responses were evaluated based on nonsensical answers. The study protocol was approved by the Institutional Review Board at the supporting institution.

\section{Analytic Strategy}

Analyses were conducted using SPSS version 25. Sample descriptive statistics and zero-order correlations among study variables were examined. Three separate, two-step hierarchical regressions were conducted for each of the criterion variables: (1) fatigue severity; (2) pain intensity; and (3) pain disability. For all analyses, step 1 covariates included years living in the U.S., COVID-19 impact, gender identity (Ramos et al., 2020), age (Lautenbacher et al., 2017), education (Bakhshaie et al., 2017), number of health conditions (Qi et al., 2020), work life distress, and home life distress (Zerbe, 2020). Step 2 included total scores on the
EA measure. Model fit for each of the steps was evaluated with the $F$ statistic and an increase in variance accounted for as evidenced by a change in $R^{2}$. Squared semi-partial correlations $\left(s r^{2}\right)$ were used as measures of effect size (interpreted as $.01=$ small, $.09=$ moderate, and $.25=$ large) (Cohen, 1988). A Bonferroni correction was employed; level of statistical significance was adjusted to .017 (i.e., .05/3).

\section{Results}

Zero-order relations and descriptive statistics are presented in Table 1. Regression results are presented in Table 2. In terms of fatigue severity, step one of the model with covariates was statistically significant $\left[R^{2}=.28, F(8,167)=7.54\right.$, $p<.001]$ with COVID impact and number of health conditions emerging as statistically significant predictors. In step two, EA resulted in a statistically significant model $\left(\Delta R^{2}=.32, p<.001\right)$.

In terms of pain intensity, step one of the model with covariates was statistically significant $\left[R^{2}=.43, F(8\right.$, $167)=17.03, p<.001]$ with COVID impact, home distress, and number of health conditions emerging as statistically significant predictors. In step two, EA resulted in a statistically significant model $\left(\Delta R^{2}=.11, p<.001\right)$.

In terms of pain disability, step one of the model with covariates was statistically significant $\left[R^{2}=.36, F(8\right.$, $167)=11.20, p<.001]$ with COVID impact, gender, health conditions, and home distress emerging as statistically significant predictors. In step two, EA remained a statistically significant predictor $\left(\Delta R^{2}=.15, p<.001\right)$.

\section{Discussion}

The current study examined EA as an individual difference factor of potential importance to better understand fatigue severity, pain intensity, and pain disability during COVID19 among Latinx adults. In line with expectation, EA was significantly related to fatigue severity, pain intensity, and pain disability. These significant associations were evident over and above the variance accounted for by years living in the U.S., COVID-19 impact, gender identity, age, education, number of health conditions, work life distress, and home life distress. Further, examination indicates the magnitude of these relations were clinically meaningful, with EA accounting for $11-32 \%$ of the (incremental) variance across criterion variables.

The current findings help bridge previous studies that highlight the importance of EA in terms of behavioral and mental health among the Latinx population in pre-pandemic research (Bakhshaie et al., 2017; Raines et al., 2018; Zvolensky et al., 2020b). These results provide initial empirical 


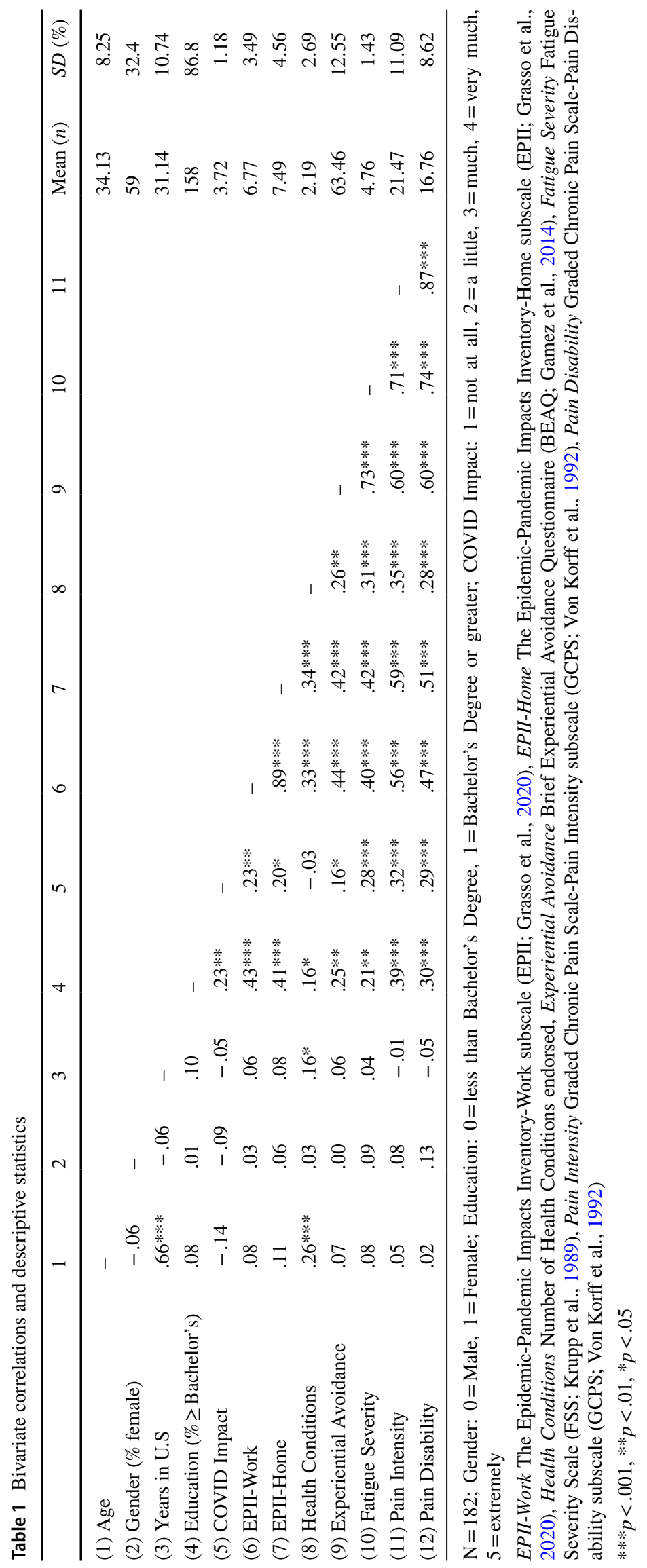


Table 2 Hierarchical regression results

\begin{tabular}{|c|c|c|c|c|c|c|c|c|}
\hline \multicolumn{9}{|c|}{ Fatigue Severity } \\
\hline Model & & $\Delta R^{2}$ & $b$ & $S E$ & $\beta$ & $t$ & $p$ & $s r^{2}$ \\
\hline \multirow[t]{8}{*}{1} & Gender & $.28 * * *$ & 0.31 & 0.21 & .10 & 1.46 & .15 & .01 \\
\hline & Years in U.S & & -0.01 & 0.01 & -.05 & -0.56 & .58 & .001 \\
\hline & Age & & 0.01 & 0.02 & .06 & 0.63 & .53 & .002 \\
\hline & Education & & -0.07 & 0.32 & -.02 & -0.22 & .82 & .0002 \\
\hline & COVID-19 Impact & & 0.32 & 0.09 & .26 & 3.62 & $<.001$ & .06 \\
\hline & EPII-Work & & 0.03 & 0.06 & .06 & 0.40 & .69 & .001 \\
\hline & EPII-Home & & 0.08 & 0.05 & .25 & 1.65 & .10 & .01 \\
\hline & Health Conditions & & 0.11 & 0.04 & .20 & 2.73 & $<.01$ & .03 \\
\hline 2 & Experiential Avoidance & $.32 * * *$ & 0.07 & 0.01 & .64 & 11.22 & $<.001$ & .32 \\
\hline \multicolumn{9}{|c|}{ Pain Intensity } \\
\hline Model & & $\Delta R^{2}$ & $b$ & $S E$ & $\beta$ & $t$ & $p$ & $s r^{2}$ \\
\hline \multirow[t]{8}{*}{1} & Gender & $.43 * * *$ & 2.00 & 1.40 & .08 & 1.43 & .16 & .01 \\
\hline & Years in U.S & & -0.16 & 0.09 & -.15 & -1.84 & .07 & .01 \\
\hline & Age & & 0.10 & 0.11 & .08 & 0.93 & .35 & .003 \\
\hline & Education & & 4.17 & 2.14 & .13 & 1.95 & .05 & .01 \\
\hline & COVID-19 Impact & & 2.20 & 0.59 & .23 & 3.74 & $<.001$ & .05 \\
\hline & EPII-Work & & 0.09 & 0.42 & .03 & 0.22 & .83 & .0002 \\
\hline & EPII-Home & & 0.98 & 0.32 & .40 & 3.10 & $<.01$ & .03 \\
\hline & Health Conditions & & 0.82 & 0.27 & .20 & 3.07 & $<.01$ & .03 \\
\hline 2 & Experiential Avoidance & $.11 * * *$ & 0.34 & 0.05 & .38 & 6.46 & $<.001$ & .11 \\
\hline \multicolumn{9}{|c|}{ Pain Disability } \\
\hline Model & & $\Delta R^{2}$ & $b$ & $S E$ & $\beta$ & $t$ & $p$ & $s r^{2}$ \\
\hline \multirow[t]{8}{*}{1} & Gender & $.36 * * *$ & 2.54 & 1.19 & .14 & 2.14 & .03 & .02 \\
\hline & Years in U.S & & -0.12 & 0.07 & -.15 & -1.66 & .10 & .01 \\
\hline & Age & & 0.06 & 0.09 & .06 & 0.67 & .51 & .002 \\
\hline & Education & & 1.73 & 1.81 & .07 & 0.96 & .34 & .004 \\
\hline & COVID-19 Impact & & 1.76 & 0.50 & .24 & 3.54 & $<.01$ & .05 \\
\hline & EPII-Work & & -0.18 & 0.36 & -.07 & -0.51 & .61 & .001 \\
\hline & EPII-Home & & 0.86 & 0.27 & .45 & 3.21 & $<.01$ & .04 \\
\hline & Health Conditions & & 0.49 & 0.23 & .15 & 2.17 & .03 & .02 \\
\hline 2 & Experiential Avoidance & $.15^{* * *}$ & 0.31 & 0.04 & .44 & 7.00 & $<.001$ & .15 \\
\hline
\end{tabular}

$\mathrm{N}=182$; Gender: $0=$ Male, $1=$ Female; Education: $0=$ less than Bachelor's Degree, $1=$ Bachelor's Degree or greater; COVID Impact: $1=$ not at all, $2=$ a little, $3=$ much, $4=$ very much, $5=$ extremely

EPII-Work The Epidemic-Pandemic Impacts Inventory-Work subscale (EPII; Grasso et al., 2020), EPII-Home The Epidemic-Pandemic Impacts Inventory-Home subscale (EPII; Grasso et al., 2020), Health Conditions Number of Health Conditions endorsed; Experiential Avoidance Brief Experiential Avoidance Questionnaire (BEAQ; Gamez et al., 2014), Fatigue Severity Fatigue Severity Scale (FSS; Krupp et al., 1989), Pain Intensity Graded Chronic Pain Scale-Pain Intensity subscale (GCPS; Von Korff et al., 1992), Pain Disability Graded Chronic Pain Scale-Pain Disability subscale (GCPS; Von Korff et al., 1992)

$* * * p<.001, * * p<.01, * p<.05$

evidence that EA may be an important individual difference factor for better understanding the experience of fatigue and pain in the context of COVID-19 among Latinx adults. Theoretically, repeated attempts to escape or avoid aversive somatic symptoms during a large-scale disaster, such as the COVID-19 pandemic, may exacerbate the severity of symptoms and distress related to such bodily perturbation
(Aldrich et al., 2000). Thus, while situational specific scape/ avoidance of an identified threat is certainly adaptative (Eippert et al., 2007), the current data and past work suggests EA, which reflects a persistent avoidance of distressing internal experiences and contexts, is a dysfunctional psychological process. Indeed, in the current study, EA demonstrated clear incremental predictive utility after adjusting 
for the emotional impact of COVID-19, suggesting that how Latinx persons respond to internal stimuli (i.e., accepting vs. avoidant) may influence health-related processes relative to the severity of COVID-19 distress. This does not mean in any way that Latinx persons are the only group wherein EA is relevant. In fact, EA should be theoretically relevant to persons of different races/ethnicities. Yet, building from this work, one logical next step in this line of research will be to examine the mediating role of EA in improvements in pain and fatigue for Latinx and other persons seeking treatment for such health problems in context of COVID-19 and its aftermath.

Although not primary study objectives, several additional observations warrant brief comment. First, the sample was characterized by a high degree of physical health problems. Moreover, such health conditions were related to each of the criterion variables, as has been evident in past work (Orwelius et al., 2010; Weijman et al., 2004). Second, COVID-19 impact was associated with the criterion variables in the regression models, adding to past work linking the pandemic experience to worse physical health (Koppert et al., 2021). Third, being female was related to greater pain disability; a finding that is in line with previous research (Bartley \& Fillingim, 2013).

Clinically, the current findings suggest that intervention strategies aimed at lowering EA (e.g., behavioral skills training to reduce avoidant behavior) may help to reduce the physical distress in the context of COVID-19 among Latinx adults. Acceptance and Commitment Therapy (ACT; Hayes et al., 2011) expressly targets EA and is efficacious for problems such as pain and chronic illness (A-tjak et al., 2015; Dindo, 2015; Hann \& McCracken, 2014). ACT has been successfully disseminated in primary care settings, which is often the context wherein Latinx persons seek help for physical and mental health problems (Aguilera et al., 2018). To the best of our knowledge, however, ACT has not been evaluated in the context of COVID-19 among the Latinx population. Given the lingering effects of COVID19 (e.g., long haulers; Rubin, 2020) and new viral variants, future research examining the utility of ACT or culturally adapted versions of ACT for the Latinx population may help persons struggling with elevated pain or fatigue (as a consequence of the COVID-19 pandemic demands or infection; Rogers et al., 2020; Zerbe, 2020) learn more adaptive ways of responding to aversive internal experiences. Further, in considering the role of EA among Latinx persons, it may be important to address cultural attitudes toward pain and fatigue symptoms (e.g., stoicism, which represents a form of ignoring pain symptoms; Torres et al., 2017).

There are limitations to the current study that warrant comment. First, the data are cross-sectional. Thus, we cannot isolate the directionality or causal nature of relations between the studied variables. Future work could employ prospective designs to more fully explore whether EA predicts greater pain and fatigue symptoms over time. Second, these data were collected via an online platform to comply with social distancing guidelines because of pandemic related restrictions on in person research and all materials were presented in English. Therefore, the sample may represent a Latinx group that has an "average to high" comfort with technology and those with English proficiency. In fact, the sample included more than 50\% reporting having attained a bachelor's degree or higher, which compares to approximately $11 \%$ among general population (Latinx samples; Schak \& Nichols, 2017). As such, the current sample may not be fully representative of the Latinx population. The generalizability of the current work is further limited as the sample was comprised of 182 individuals and only $32.4 \%$ of the cohort were female. Thus, this sample may not be adequately sized nor balanced by sex to fully represent the general Latinx pupation. Research indicates that while males participate at higher rates than females on informational online platforms, females are more present on social media platforms; therefore, future work would benefit from recruiting on social media platforms as well to include more female participation (Sun et al., 2020; Vasilescu et al., 2014). Third, no behavioral measures of pain or fatigue were included. Future work may seek to implement a multimethod approach to assess fatigue and pain among Latinx persons (e.g., experimental pain assessment). Fourth, no information on pre-COVID fatigue and pain symptoms were measured; thus, it is not clear whether EA may have contributed to onset or exacerbation of these symptoms. Moreover, the current study did not assess whether fatigue and pain were related to COVID-19 infection. The question therefore of whether observed relations hold or are more apparent among those with post or current COVID-19 infection warrants further investigation. Fifth, the current analyses were conducted on a general population sample of Latinx persons. Future work would benefit from evaluating these relations within a sample of persons with chronic pain and clinically significant fatigue. Finally, although we modeled EA and adjusted for the stress related to COVID-19, future research could replicate and extend these findings to independent Latinx samples and adjust for other higher-order negative emotional traits (e.g., negative affectivity). Further, there would be value in exploring other vulnerability factors (e.g., rumination, worry) and protective factors (e.g., adaptive coping, resiliency) for pain and fatigue among the Latinx population in the context of the ongoing pandemic.

Overall, the current findings provide initial empirical evidence for the role of EA as a psychological factor that is related to greater fatigue severity and pain severity and disability among Latinx persons during COVID-19. Future research using a prospective design is needed to understand the interplay between EA and fatigue and pain severity and 
disability would be useful for this underserved and overrepresented minority group in terms of clinical correlates of COVID-19.

Funding Research reported in this publication was supported by the National Institute on Minority Health and Health Disparities (NIMHD) of the National Institutes of Health (NIH) to the University of Houston under Award Number U54MD015946. The content is solely the responsibility of the authors and does not necessarily represent the official views of the National Institutes of Health. This work was also supported by the National Institute of Drug Abuse (F31 DA051199).

\section{Declarations}

Conflict of Interest Nubia A. Mayorga, Kara F. Manning, Lorra Garey, Andres G. Viana, Joseph W. Ditre, and Michael J. Zvolensky declare that they have no conflict of interest.

Ethical Approval All procedures performed in studies involving human participants were in accordance with the ethical standards of the Institutional and/or National Research Committee and with the 1964 Helsinki Declaration and its later amendments or comparable ethical standards.

Informed Consent Informed consent was obtained from all individual participants included in the study.

\section{References}

Aguilera, A., Bruehlman-Senecal, E., Liu, N., \& Bravin, J. (2018). Implementing group CBT for depression among Latinos in a primary care clinic. Cognitive and Behavioral Practice, 25(1), 135-144.

Aldrich, S., Eccleston, C., \& Crombez, G. (2000). Worrying about chronic pain: Vigilance to threat and misdirected problem solving. Behaviour Research and Therapy, 38(5), 457-470.

A-tjak, J. G., Davis, M. L., Morina, N., Powers, M. B., Smits, J. A., \& Emmelkamp, P. M. (2015). A meta-analysis of the efficacy of acceptance and commitment therapy for clinically relevant mental and physical health problems. Psychotherapy and Psychosomatics, 84(1), 30-36.

Bakhshaie, J., Kauffman, B. Y., Viana, A. G., Garza, M., Ochoa-Perez, M., Lemaire, C., Bogiaizian, D., Robles, Z., \& Zvolensky, M. J. (2017). Synergistic effects of pain intensity and experiential avoidance in relation to anxiety symptoms and disorders among economically disadvantaged Latinos in a community-based primary care setting. Journal of Anxiety Disorders, 48, 54-62.

Bakhshaie, J., Rogers, A. H., Mayorga, N. A., Ditre, J., RodríguezCano, R., Ruiz, A. C., Viana, A. G., Garza, M., Lemaire, C., \& Ochoa-Perez, M. (2019). Perceived racial discrimination and pain intensity/disability among economically disadvantaged Latinos in a Federally Qualified Health Center: The role of anxiety sensitivity. Journal of Immigrant and Minority Health, 21(1), 21-29.

Bakshi, R., Miletich, R. S., Henschel, K., Shaikh, Z. A., Janardhan, V., Wasay, M., Stengel, L. M., Ekes, R., \& Kinkel, P. R. (1999). Fatigue in multiple sclerosis: Cross-sectional correlation with brain MRI findings in 71 patients. Neurology, 53(5), 1151-1151.

Bartley, E. J., \& Fillingim, R. B. (2013). Sex differences in pain: A brief review of clinical and experimental findings. British Journal of Anaesthesia, 111(1), 52-58.
Campbell, L. C., Andrews, N., Scipio, C., Flores, B., Feliu, M. H., \& Keefe, F. J. (2009). Pain coping in Latino populations. The Journal of Pain, 10(10), 1012-1019.

CDC. (2020, February 11). Coronavirus Disease 2019 (COVID-19). Centers for Disease Control and Prevention. https://www.cdc.gov/ coronavirus/2019-ncov/covid-data/investigations-discovery/hospi talization-death-by-race-ethnicity.html

Chen, M. K. (1986). The epidemiology of self-perceived fatigue among adults. Preventive Medicine, 15(1), 74-81.

Cohen, J. (1988). Statistical power analysis for the behavioral sciences $\left(2^{\text {nd }}\right.$ ed.). Lawrence Erlbaum Associates.

COVID-19 provisional counts—Health disparities (2021, February 3 ). https://www.cdc.gov/nchs/nvss/vsrr/covid19/health_disparities. htm

Dindo, L. (2015). One-day acceptance and commitment training workshops in medical populations. Current Opinion in Psychology, $2,38-42$.

Eippert, F., Veit, R., Weiskopf, N., Erb, M., Birbaumer, N., \& Anders, S. (2007). Regulation of emotional responses elicited by threatrelated stimuli. Human Brain Mapping, 28(5), 409-423.

Feldner, M. T., Zvolensky, M. J., Babson, K., Leen-Feldner, E. W., \& Schmidt, N. B. (2008). An integrated approach to panic prevention targeting the empirically supported risk factors of smoking and anxiety sensitivity: Theoretical basis and evidence from a pilot project evaluating feasibility and short-term efficacy. Journal of Anxiety Disorders, 22(7), 1227-1243.

Gamez, W., Chmielewski, M., Kotov, R., Ruggero, C., \& Watson, D. (2014). Development of a measure of experiential avoidance: The Multidimensional Experiential Avoidance Questionnaire (MEAQ). Psychological Assessment, 23(3), 692-713.

Grasso, D. J., Briggs-Gowan, M. J., Carter, A. S., Goldstein, B., \& Ford, J. D. (2020). A person-centered approach to profiling COVID-related experiences in the United States: Preliminary findings from the Epidemic-Pandemic Impacts Inventory (EPII). PsyArXiv. https://doi.org/10.31234/osf.io/v36hj

Hann, K. E., \& McCracken, L. M. (2014). A systematic review of randomized controlled trials of Acceptance and Commitment Therapy for adults with chronic pain: Outcome domains, design quality, and efficacy. Journal of Contextual Behavioral Science, 3(4), 217-227.

Hayes, S. C., Strosahl, K. D., \& Wilson, K. G. (2011). Acceptance and commitment therapy: The process and practice of mindful change. Guilford Press.

Hayes, S. C., Strosahl, K., Wilson, K. G., Bissett, R. T., Pistorello, J., Toarmino, D., Polusny, M. A., Dykstra, T. A., Batten, S. V., \& Bergan, J. (2004). Measuring experiential avoidance: A preliminary test of a working model. The Psychological Record, 54(4), 553-578.

Hayes, S. C., Wilson, K. G., Gifford, E. V., Follette, V. M., \& Strosahl, K. (1996). Experiential avoidance and behavioral disorders: A functional dimensional approach to diagnosis and treatment. Journal of Consulting and Clinical Psychology, 64(6), 1152.

Jacobson, N. S., Martell, C. R., \& Dimidjian, S. (2001). Behavioral activation treatment for depression: Returning to contextual roots. Clinical Psychology: Science and Practice, 8(3), 255-270.

Jason, L. A., Jordan, K. M., Richman, J. A., Rademaker, A. W., Huang, C.-F., Mccready, W., Shlaes, J., King, C. P., Landis, D., \& Torres, S. (1999). A community-based study of prolonged fatigue and chronic fatigue. Journal of Health Psychology, 4(1), 9-26.

Kemp, H. I., Corner, E., \& Colvin, L. A. (2020). Chronic pain after COVID-19: Implications for rehabilitation. British Journal of Anaesthesia, 125(4), 436-440.

Kim, H. S., \& Hodgins, D. C. (2017). Reliability and validity of data obtained from alcohol, cannabis, and gambling populations on Amazon's Mechanical Turk. Psychology of Addictive Behaviors, 31(1), 85. 
Koppert, T. Y., Jacobs, J. W., Lumley, M. A., \& Geenen, R. (2021). The impact of COVID-19 stress on pain and fatigue in people with and without a central sensitivity syndrome. Journal of Psychosomatic Research, 151, 110655.

Krupp, L. B., LaRocca, N. G., Muir-Nash, J., \& Steinberg, A. D. (1989). The fatigue severity scale: Application to patients with multiple sclerosis and systemic lupus erythematosus. Archives of Neurology, 46(10), 1121-1123.

Kwok, W., \& Bhuvanakrishna, T. (2014). The relationship between ethnicity and the pain experience of cancer patients: A systematic review. Indian Journal of Palliative Care, 20(3), 194.

Lautenbacher, S., Peters, J. H., Heesen, M., Scheel, J., \& Kunz, M. (2017). Age changes in pain perception: A systematic-review and meta-analysis of age effects on pain and tolerance thresholds. Neuroscience and Biobehavioral Reviews, 75, 104-113.

Lawrence, E. M. (2017). Why do college graduates behave more healthfully than those who are less educated? Journal of Health and Social Behavior, 58(3), 291-306.

Lerdal, A., Wahl, A. K., Rustoen, T., Hanestad, B. R., \& Moum, T. (2005). Fatigue in the general population: A translation and test of the psychometric properties of the Norwegian version of the fatigue severity scale. Scandinavian Journal of Public Health, 33(2), 123-130.

Loge, J. H., Ekeberg, Ø., \& Kaasa, S. (1998). Fatigue in the general Norwegian population: Normative data and associations. Journal of Psychosomatic Research, 45(1), 53-65.

Lovell, N., Maddocks, M., Etkind, S. N., Taylor, K., Carey, I., Vora, V., Marsh, L., Higginson, I. J., Prentice, W., \& Edmonds, P. (2020). Characteristics, symptom management, and outcomes of 101 patients with COVID-19 referred for hospital palliative care. Journal of Pain and Symptom Management, 60(1), e77-e81.

Manning, K., Garey, L., Mayorga, N. A., Shepherd, J. M., \& Zvolensky, M. J. (2019). The relation between fatigue severity and anxious arousal, negative affect, and emotion dysregulation among adult e-cigarette users. Fatigue: Biomedicine, Health and Behavior, $7(2), 92-101$.

McCracken, L. M., \& Eccleston, C. (2003). Coping or acceptance: What to do about chronic pain? Pain, 105(1-2), 197-204.

McKinney, E. F., Lee, J. C., Jayne, D. R., Lyons, P. A., \& Smith, K. G. (2015). T-cell exhaustion, co-stimulation and clinical outcome in autoimmunity and infection. Nature, 523(7562), 612-616.

Meichtry, S., Sugden, J., \& Barnett, A. (2020). Pandemic fatigue is real... and it's spreading. Wall Street Journal. www.wsj.com

Morgul, E., Bener, A., Atak, M., Akyel, S., Aktaş, S., Bhugra, D., Ventriglio, A., \& Jordan, T. R. (2020). COVID-19 pandemic and psychological fatigue in Turkey. The International Journal of Social Psychiatry, 67(2), 128-135.

Orwelius, L., Nordlund, A., Nordlund, P., Simonsson, E., Bäckman, C., Samuelsson, A., \& Sjöberg, F. (2010). Pre-existing disease: The most important factor for health related quality of life long-term after critical illness: A prospective, longitudinal, multicentre trial. Critical Care, 14(2), 1-10.

Paulus, D. J., Viana, A. G., Ditre, J. W., Bakhshaie, J., Garza, M., Berger Cardoso, J., Valdivieso, J., Ochoa-Perez, M., Lemaire, C., \& Zvolensky, M. J. (2017). Synergistic effects of pain and alcohol use in relation to depressive and anxiety symptoms among Latinos in primary care. Cognitive Behaviour Therapy, 46(6), 478-492.

Qi, R., Chen, W., Liu, S., Thompson, P. M., Zhang, L. J., Xia, F., Cheng, F., Hong, A., Surento, W., \& Luo, S. (2020). Psychological morbidities and fatigue in patients with confirmed COVID-19 during disease outbreak: Prevalence and associated biopsychosocial risk factors. MedRxiv. https://doi.org/10.1101/2020.05.08. 20031666

Raines, E. M., Rogers, A. H., Bakhshaie, J., Viana, A. G., Lemaire, C., Garza, M., Mayorga, N. A., Ochoa-Perez, M., \& Zvolensky, M. J. (2018). Mindful attention moderating the effect of experiential avoidance in terms of mental health among Latinos in a federally qualified health center. Psychiatry Research, 270, 574-580.

Ramos, A. K., Carvajal-Suarez, M., Trinidad, N., Michaud, T. L., Grimm, B., LeVan, T., \& Siahpush, M. (2020). A cross-sectional study of gender-related differences in reporting fatigue and pain among Latino/a migrant farmworkers. Journal of Agromedicine, 25(3), 1-11.

Resnick, A., Galea, S., \& Sivashanker, K. (2020). COVID-19: The painful price of ignoring health inequities. BMJ Opinion. https:// blogs.bmj.com/bmj/2020/03/18/covid-19-the-painful-price-ofignoring-health-inequities/

Reyes-Gibby, C. C., Anderson, K. O., Shete, S., Bruera, E., \& Yennurajalingam, S. (2012). Early referral to supportive care specialists for symptom burden in lung cancer patients: A Comparison of Non-Hispanic Whites, Hispanics, and Non-Hispanic Blacks. Cancer, 118(3), 856-863.

Rogers, A. H., Garey, L., \& Zvolensky, M. J. (2021). COVID-19 psychological factors associated with pain status, pain intensity, and pain-related interference. Cognitive Behaviour Therapy, 50(6), $1-13$.

Rogers, A. H., Shepherd, J. M., Garey, L., \& Zvolensky, M. J. (2020). Psychological factors associated with substance use initiation during the COVID-19 pandemic. Psychiatry Research, 293, 113407.

Rubin, R. (2020). As their numbers grow, COVID-19 "long haulers" stump experts. JAMA, 324(14), 1381-1383.

Schak, J. O., \& Nichols, A. H. (2017). Degree attainment for Latino adults: National and state trends (p. 11). Education Trust.

Siebenhaar, K. U., Köther, A. K., \& Alpers, G. W. (2020). Dealing with the COVID-19 infodemic: Distress by information, information avoidance, and compliance with preventive measures. Frontiers in Psychology, 11, 2981.

Song, S., Jason, L. A., \& Taylor, R. R. (1999). The relationship between ethnicity and fatigue in a community-based sample. Journal of Gender, Culture and Health, 4(4), 255-268.

Sprang, G., \& LaJoie, A. S. (2009). Exposure, avoidance, and PTSD among Hurricane Katrina evacuees. Traumatology, 15(2), 10-19.

Sun, B., Mao, H., \& Yin, C. (2020). Male and female users' differences in online technology community based on text mining. Frontiers in Psychology, 11, 806.

Too exhausted to fight, immune system may harm the body they are supposed to protect-ScienceDaily (n.d.). Retrieved March 12, 2021, from https://www.sciencedaily.com/releases/2015/06/15062 9110803.htm

Torres, C. A., Thorn, B. E., Kapoor, S., \& DeMonte, C. (2017). An examination of cultural values and pain management in foreignborn Spanish-speaking Hispanics seeking care at a federally qualified health center. Pain Medicine, 18(11), 2058-2069.

Torres-Harding, S. R., Mason-Shutter, J., \& Jason, L. A. (2008). Fatigue among Spanish- and English-speaking Latinos. Social Work in Public Health, 23(5), 55-72.

Van Damme, S., Crombez, G., Van Houdenhove, B., Mariman, A., \& Michielsen, W. (2006). Well-being in patients with chronic fatigue syndrome: The role of acceptance. Journal of Psychosomatic Research, 61(5), 595-599.

Vasilescu, B., Capiluppi, A., \& Serebrenik, A. (2014). Gender, representation and online participation: A quantitative study. Interacting with Computers, 26(5), 488-511.

Velasco-Mondragon, E., Jimenez, A., Palladino-Davis, A. G., Davis, D., \& Escamilla-Cejudo, J. A. (2016). Hispanic health in the USA: A scoping review of the literature. Public Health Reviews, 37(1), $1-27$.

Von Korff, M., Ormel, J., Keefe, F. J., \& Dworkin, S. F. (1992). Grading the severity of chronic pain. Pain, 50(2), 133-149.

Weijman, I., Kant, Ij., Swaen, G. M., Ros, W. J., Rutten, G. E., Schaufeli, W. B., Schabracq, M. J., \& Winnubst, J. A. (2004). Diabetes, employment and fatigue-related complaints: A comparison 
between diabetic employees, "healthy" employees, and employees with other chronic diseases. Journal of Occupational and Environmental Medicine, 46(8), 828-836.

WHO Coronavirus Disease (COVID-19) Dashboard (n.d.). Retrieved November 1, 2020, from https://covid19.who.int

Who-china-joint-mission-on-covid-19-final-report.pdf (2020). Retrieved February 10, 2021, from https://www.who.int/docs/ default-source/coronaviruse/who-china-joint-mission-on-covid19-final-report.pdf

Wright, J., \& O'Connor, K. M. (2014). Fatigue. Medical Clinics of North America, 98(3), 597-608. https://doi.org/10.1016/j.mcna. 2014.01.010

Zerbe, K. J. (2020). Pandemic fatigue: Facing the body's inexorable demands in the time of COVID-19. Journal of the American Psychoanalytic Association, 68(3), 475-478.

Zvolensky and J., Garey, L., Rogers, A. H., Schmidt, N. B., Vujanovic, A. A., Storch, E. A., Buckner, J. D., Paulus, D. J., Alfano, C. A.,
Smits, J. A., \& O'Cleirigh, C. (2020a). Psychological, addictive, and health behavior implications of the COVID-19 pandemic. Behaviour Research and Therapy. https://doi.org/10.1016/j.brat. 2020.103715

Zvolensky, M. J., Garey, L., Rogers, A. H., Schmidt, N. B., Vujanovic, A. A., Storch, E. A., Buckner, J. D., Paulus, D. J., Alfano, C., \& Smits, J. A. (2020b). Psychological, addictive, and health behavior implications of the COVID-19 pandemic. Behaviour Research and Therapy, 134, 103715.

Publisher's Note Springer Nature remains neutral with regard to jurisdictional claims in published maps and institutional affiliations. 